\title{
CALCULATING INVARIANTS OF INSEPARABLE FIELD EXTENSIONS
}

\author{
JAMES K. DEVENEY AND JOHN N. MORDESON
}

\begin{abstract}
Let $L$ be a finitely generated nonalgebraic extensions of a field $K$ of characteristic $p \neq 0$ and let $M$ be a finite purely inseparable extension of $L$. This paper is concerned with calculating inseparability-related numerical invariants of $M / K$ from those of $L / K$.
\end{abstract}

I. Introduction. Let $L$ be a finitely generated field extension of a field $K$ of characteristic $p \neq 0$. If $D$ is a maximal separable extension of $K$ in $L$, then $L$ is purely inseparable finite dimensional over $D$. If $p^{s}$ is the smallest of the dimensions of $L$ over such maximal separable extensions, then $s$ is Weil's order of inseparability of $L / K($ denoted inor $(L / K))$ [7]. If $[L: D]$ is minimal, then $L \subseteq K^{p^{-\infty}}(D)$ and $D$ is called distinguished for $L / K$ [3], [6]. There are two other important invariants of $L / K$ which were introduced in [6], the inseparability exponent of $L / K$, $\operatorname{inex}(L / K)=\min \left\{r \mid K\left(L^{p^{\prime}}\right)\right.$ is separable over $\left.K\right\}$ and the inseparability of $L / K$, insep $(L / K)=\log _{p}\left[L: K\left(L^{p}\right)\right]-$ transcendence degree of $L / K$. These are related, for example inex $(L / K)=\min \left\{r \mid K\left(L^{p^{\prime}}\right)=K\left(D^{p^{\prime}}\right)\right.$ for a distinguished $\left.D\right\}=$ $\min \left\{r \mid L \subseteq K^{p^{-r}}(D)\right.$ for a distinguished $\left.D\right\}$ [5, Proposition 1, p. 288].

This paper is concerned with the following question. Given $D$, a finitely generated separable extension of $K$, and $L=D\left(c_{1}, c_{2}, \ldots, c_{m}\right)$, where each $c_{i}$ is purely inseparable over $D$, how can one calculate inor $(L / K)$, inex $(L / K)$ and insep $(L / K)$ ? There are two main tools which will be used. First are the fields $K\left(L^{(r)}\right)=\left\{x \in L \mid x^{p^{j}} \in K\left(L^{p^{r+}}\right)\right.$ for some $\left.j>0\right\}$ which were studied in [5]. $L=$ $K\left(L^{(0)}\right) \supseteq K\left(L^{(1)}\right) \supseteq K\left(L^{(2)}\right) \supseteq \cdots \quad$ and $K\left(L^{(\infty)}\right)$ will denote $\cap, K\left(L^{(r)}\right)$. Recall that $K\left(L^{(\infty)}\right)$ is the algebraic closure of $K$ in $L$ [5, Theorem 5, p. 289]. Also note that $\cap, K\left(L^{p^{\prime}}\right) \equiv K\left(L^{\infty}\right)$ is the separable algebraic closure of $K$ in $L$ [4, Theorem 7.2, p. 273]. The second main tool is the concept of a form. An intermediate field $F$ of $L / K$ is a form of $L / K$ if and only if $\operatorname{inor}(L / K)=\operatorname{inor}(F / K)$. Forms were first studied in [2]. Every finitely generated $L / K$ has a unique minimal form $L^{\prime}$, $L \supseteq L^{\prime} \supseteq K$. The results established here can be used to determine properties of $L \supseteq L^{\prime} \supseteq K$ and to construct examples with proper forms.

We introduce some notation which will be used to state the main results. $L=D\left(c_{1}, c_{2}, \ldots, c_{m}\right)$ where $D$ is finitely generated separable over $K$ and each $c_{i}$ is purely inseparable over $D$. We define $L_{i}=D\left(c_{1}, c_{2}, \ldots, c_{i}\right) ; L_{0}=D ; p^{e_{i}}=$ $\left[L_{i}: L_{i-1}\right] ; n_{i}=\operatorname{inex}\left(L_{i} / K\right) ; c_{i}^{p_{i}} \in L_{i-1}$, and $r_{i}=\max \left\{t \mid c_{i}^{p^{c_{i}}} \in K\left(L_{i-1}^{(t)}\right)\right\}$ if the

Received by the editors February 10, 1980; presented to the Society, April 18, 1980.

1980 Mathematics Subject Classification. Primary 12F15.

Key words and phrases. Order of inseparability, distinguished subfield.

(c) 1981 American Mathematical Society 0002-9939/81/0000-0106/\$02.00 
maximum exists and is $\infty$ otherwise; $j_{i}=\min \left\{\mu \mid c_{i}^{p_{i}+\mu} \in K\left(L_{i-1}^{p_{i+\mu}+\mu}\right)\right\}$ if $r_{i}<\infty$ and $j_{i}=\min \left\{\mu \mid c_{i}^{p_{i}+\mu} \in K\left(L^{\infty}\right)\right\}$ if $r_{i}=\infty$. (We note that if $r_{i}=\infty$, then $c_{i}$ is algebraic over $K$ and since $K\left(L^{\infty}\right)$ is the separable algebraic closure of $K$ in $L$, $e_{i}+j_{i}$ is the least $p$ th power of $c_{i}$ which is separable over $K$.) The main results on the stated problem are

$$
\begin{gathered}
\operatorname{inor}(L / K)=\sum_{i=1}^{m} \min \left\{e_{i}, r_{i}\right\} ; \\
\operatorname{inex}(L / K)=\max \left\{q_{1}+j_{1}, \ldots, q_{m}+j_{m}\right\}, \quad q_{i}=\min \left\{e_{i}, r_{i}\right\} ; \\
\operatorname{insep}(L / K)=d
\end{gathered}
$$

where $d$ is the number of $c_{i}$ such that $c_{i}^{p_{i}} \in K\left(L_{i-1}^{p}\right)$.

II. The first result is essentially contained in [2], but is presented here for clarity. It gives an exponent-independent characterization of a form of $L / K$.

Proposition 0. Let $L$ be a finitely generated extension of $K$ and let $L_{1}$ be an intermediate field. Then $L_{1}$ is a form of $L / K$ if and only if $L^{p^{r}}$ and $K\left(L_{1}^{p^{\prime}}\right)$ are linearly disjoint over $L_{1}^{p^{r}}$ for all $r$.

Proof. Let $n$ be the exponent of $L$ over $K$. If we have linear disjointness for all $r$, then we certainly have it for $r=n$, so $L_{1}$ is a form of $L / K$ [2, Theorem 1.3, p. 656]. Assume we have disjointness for $n$. If $r<n$, then since $K\left(L_{1}^{p^{n}}\right) \supset K^{p^{n-1}}\left(L_{1}^{p^{n}}\right) \supset$ $L_{1}^{p^{n}}, L^{p^{n}}$ and $K^{p^{n-r}}\left(L_{1}^{p^{n}}\right)$ are linearly disjoint over $L_{1}^{p^{n}}$. Taking $p^{n-r}$ th roots of these fields gives the desired linear disjointness. If $r>n$, by induction it suffices to prove the case $r=n+1$. Taking $p$ th roots, we need $L^{p^{n}}$ and $K^{p^{-1}}\left(L_{1}^{p^{n}}\right)$ linearly disjoint over $L_{1}^{p^{n}}$. But since $K^{p^{-1}}\left(L_{1}^{p^{n}}\right) \supset K\left(L_{1}^{p^{n}}\right) \supset L_{1}^{p^{n}}$, by the standard lemma on linear disjointness we only need show $K\left(L^{p n}\right)$ and $K^{p^{-1}}\left(L_{1}^{p^{*}}\right)$ are linearly disjoint over $K\left(L_{1}^{p^{n}}\right)$. But this follows since $K\left(L^{p^{n}}\right)$ and $K^{p^{-1}}$ are linearly disjoint over $K$, as $K\left(L^{p n}\right)$ is separable over $K$.

LEMMA 1. Let $F$ be a finitely generated extension of $K$ and let $x \in F \backslash F^{p}$.

(1) If $x \in K\left(F^{p}\right)$, then inor $\left(F\left(x^{p^{-1}}\right) / K\right)=\operatorname{inor}(F / K)+1$.

(2) If $x \in K\left(F^{(1)}\right)$, then inor $\left(F\left(x^{p^{-1}}\right) / K\right)=\operatorname{inor}(F / K)+1$.

(3) If $x \in F \backslash K\left(F^{(1)}\right)$, then $\operatorname{inor}\left(F\left(x^{p^{-1}}\right) / K\right)=\operatorname{inor}(F / K)$.

Proof. Since $K\left(F^{p}\right) \subseteq K\left(F^{(1)}\right)$, (1) follows once we prove (2). Since $x \in K\left(F^{(1)}\right)$, $x^{p^{\prime}} \in K\left(F^{p^{\prime+1}}\right)$ for some $r$. If the conclusion of (2) were false, then $F / K$ would be a form of $F\left(x^{p^{-1}}\right) / K$. Thus by Proposition $0,\left(F\left(x^{p^{-1}}\right)\right)^{p^{r+1}}$ and $K\left(F^{p^{\prime+1}}\right)$ are linearly disjoint over $F^{p^{\prime+1}}$, and in particular have $F^{p^{r+1}}$ as their intersection. But then $x^{p^{\prime}} \in F^{p^{\prime+1}}$ and hence $x \in F^{p}$, a contradiction. Thus we have (2). For (3), $F^{p^{s}}\left(x^{p^{s-1}}\right)$ and $K\left(F^{p^{s}}\right)$ must be linearly disjoint over $F^{p^{s}}$ since $\left[F^{p^{s}}\left(x^{p^{s-1}}\right): F^{p^{s}}\right]=p$ and $x^{p^{s-1}} \notin K\left(F^{p^{s}}\right)$. Thus $F / K$ is a form of $F\left(x^{p^{-1}}\right) / K$.

TheOREM 2. Let $F$ be a finitely generated extension of $K$ and let $x \in F \backslash F^{p}$. Assume $r=\max \left\{t \mid x \in K\left(F^{(t)}\right)\right\}$ if it exists and $r=\infty$ otherwise. Then $\operatorname{inor}\left(F\left(x^{p^{-\varepsilon}}\right)\right)=\operatorname{inor}(F / K)+\min \{e, r\}, e>0$. 
Proof. The proof is by induction on $e$. If $e=1$, the result is Lemma 1 . Consider the chain of fields $F\left(x^{p^{-e}}\right) \supseteq F\left(x^{p^{-e+1}}\right) \supseteq F$. Since $\left[F\left(x^{p^{-e+1}}\right): F\right]=p^{e-1}$, $\operatorname{inor}\left(F\left(x^{p^{-e+1}}\right) / K\right)=\min \{e-1, r\}$ by induction. Thus the result will be established once we show inor $\left(F\left(x^{p^{-e}}\right) / K\right)=\operatorname{inor}\left(F\left(x^{p^{e+1}}\right) / K\right)+1$ if and only if $e<r$. Suppose $\operatorname{inor}\left(F\left(x^{p^{-e}}\right) / K\right)=\operatorname{inor}\left(F\left(x^{p^{-e+1}}\right) / K\right)+1$. Then $x^{p^{-e+1}} \in$ $K\left(\left(F\left(x^{p^{-e+1}}\right)\right)^{(1)}\right)$ by Lemma 1. Thus $\left(x^{p^{-c+1}}\right)^{p^{s}} \in K\left(\left(F\left(x^{p^{-c+1}}\right)\right)^{p+1}\right)$ for some $s$, and in fact for $s$ as large as we wish. Hence take $s>e-1$. Then $x^{p^{j}} \in K\left(F^{p^{e+1}}\right)\left(x^{p^{j+1}}\right)$ where $j=-e+1+s$. Thus $x^{p^{j}}$ is separable over $K\left(F^{p^{e+1}}\right)$. Hence $x^{p^{j}} \in K\left(F^{p^{e+j}}\right)$ and so $e<r$. Conversely, suppose $e<r<\infty$. Then $x^{p^{\prime}} \in K\left(F^{p^{e+j}}\right)$ for some $j$ and $j$ can be taken as large as we wish. Thus for $j=-e+1+s$ where $s>e-1$, $\left(x^{p^{-c+1}}\right)^{p^{s}} \in K\left(\left(F\left(x^{p^{-c+1}}\right)\right)^{p^{s+1}}\right)$. Hence $x^{p^{-c+1}} \in K\left(\left(F\left(x^{p^{-c+1}}\right)\right)^{(1)}\right)$ and so $\operatorname{inor}\left(F\left(x^{p^{-q}}\right) / K\right)=\operatorname{inor}\left(F\left(x^{p^{-e+1}}\right) / K\right)+1$ by Lemma 1. Suppose $e<r=\infty$. However, if $r=\infty$, then $x$ and hence $x^{p^{-c+1}}$ is algebraic over $K$. Thus $x^{p^{-c+1}} \in$ $K\left(\left(F\left(x^{p^{-e+1}}\right)\right)^{(\infty)}\right)$ and inor $\left(F\left(x^{p^{-\varepsilon}}\right) / K\right)=\operatorname{inor}\left(F\left(X^{p^{-e+1}}\right) / K\right)+1$ by Lemma 1.

COROllary 3. In the notation of the introduction, $\operatorname{inor}(L / K)=\sum_{i=1}^{m} \min \left\{e_{i}, r_{i}\right\}$.

Proof. This follows from $m$ applications of Theorem 2 to the chain of fields $D=L_{0} \subset L_{1} \subset \cdots \subset L_{m}=L$ and the fact that $\operatorname{inor}(D / K)=0$.

Lemma 4. Let $F$ be a finitely generated extension of $K$ and let $x \in F \backslash F^{p}$. Let $r=\max \left\{t \mid x \in K\left(F^{(t)}\right)\right\}$ if it exists and $r=\infty$ otherwise. If $e>r$, then

$$
\operatorname{inex}\left(F\left(x^{p^{-\varepsilon}}\right) / K\right)=\operatorname{inex}\left(F\left(x^{p^{-r}}\right) / K\right) \text {. }
$$

Proof. By Theorem 2, inor $\left(F\left(x^{p^{-q}}\right) / K\right)=\operatorname{inor}\left(F\left(x^{p^{-r}}\right) / K\right)=\operatorname{inor}(F / K)+r$. Thus $F\left(x^{p^{-r}}\right)$ is a form of $F\left(x^{p^{-\varepsilon}}\right)$ and hence they both have the same inseparability exponent [2, Theorem 1.3, p. 656].

TheOREM 5. Let $F$ be a finitely generated extension of $K$ and let $x \in F \backslash F^{p}$. Let $r=\max \left\{t \mid x \in K\left(F^{(t)}\right)\right\}$ if it exists and $r=\infty$ otherwise. Let $j$ be the least nonnegative integer such that $x^{p^{\prime}} \in K\left(F^{p^{r+j}}\right)$. Then

$$
\operatorname{inex}\left(F\left(x^{p^{-e}}\right) / K\right)=\max (\operatorname{inex}(F / K), \min (e, r)+j) \text {. }
$$

Proof. In view of Lemma 4, we may assume $e<r$. Let $D$ be a distinguished subfield of $F / K$ and let $t=\operatorname{inex}(F / K)$. Then $F \subseteq K^{p^{-1}}(D)$ [5, Proposition 1, p. 288]. By Theorem 2 , inor $\left(F\left(x^{p^{-e}}\right) / K\right)=\operatorname{inor}(F / K)+e$, so $D$ is also distinguished for $F\left(x^{p^{-\varepsilon}}\right) / K$. Now $x^{p^{j}} \in K\left(F^{p^{r+j}}\right)$. So $x^{p^{-e}} \in K^{p^{-e-j}}\left(F^{p^{r-\varepsilon}}\right) \subseteq K^{p^{-e-j}}(F) \subseteq$

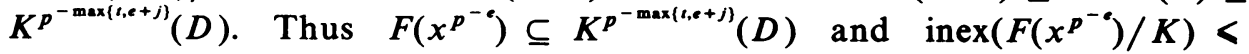
$\max \{\operatorname{inex}(F / K), e+j\}$. Now assume $F\left(x^{p^{-\varepsilon}}\right) \subseteq K^{p^{-s}}(D)$. Then clearly $s>$ inex $(F / K)$. Suppose $s<e+j<r+j$. Then $x^{p^{-e}} \in K^{p^{-s}}(D)$. Thus $\left(x^{p^{-e}}\right)^{p^{p+j}}=$ $x^{p^{j}} \in K^{p}\left(D^{p^{e+j}}\right)$ and $x^{p^{j}} \in K\left(F^{p^{r+j}}\right)$. But since $x^{p^{j}} \in D$ and $K\left(F^{p^{r+j}}\right)$ and $D \cap$ $K\left(F^{p^{r+j}}\right)=K\left(D^{p^{r+j}}\right)$ [1, Theorem 2.9, p. 1310], $x^{p^{j}} \in K^{p}\left(D^{p^{e+j}}\right) \cap K\left(D^{p^{r+j}}\right)=$ $K^{p}\left(D^{p^{r+j}}\right)$ since $D$ is separable over $K$. Thus $x^{p^{j-1}} \in K\left(D^{p^{r+j-1}}\right) \subseteq K\left(F^{p^{r+j-1}}\right)$. If $j>0$, this contradicts the definition of $j$ and if $j=0$, this contradicts the degree of $x^{p^{-e}}$ over $F$. Thus $s<e+j$ is impossible. 
COROLlaRY 6. In the notation of the introduction,

$$
\begin{gathered}
\operatorname{inex}(L / K)=\max \left\{q_{1}+j_{1}, q_{2}+j_{2}, \ldots, q_{m}+j_{m}\right\}, \\
q_{i}=\min \left\{e_{i}, r_{i}\right\} .
\end{gathered}
$$

Theorem 7. Let $F$ be a finitely generated extension of a field $K$ and let $x \in$ $F \backslash F^{p}, e>0$.

(1) If $x \notin K\left(F^{p}\right)$, insep $\left(F\left(x^{p^{-e}}\right) / K\right)=\operatorname{insep}(F / K)$.

(2) If $x \in K\left(F^{p}\right), \operatorname{insep}\left(F\left(x^{p^{-e}}\right) / K\right)=\operatorname{insep}(F / K)+1$.

Proof. (1) $x$ is $p$-independent in $F / K$. Let $\{x\} \cup C$ be a relative $p$-basis of $F / K$. Then $\left\{x^{p^{-e}}\right\} \cup C$ is a relative $p$-basis of $F\left(x^{p^{-e}}\right) / K$.

(2) Since $x \in K\left(F^{p}\right), K\left(\left(F\left(x^{p^{-\varepsilon}}\right)\right)^{p}\right)$ and $F$ are linearly disjoint over $K\left(F^{p}\right)$. Thus if $C$ is a relative $p$-basis of $F / K, C$ is also relatively $p$-independent in $F\left(x^{p^{-e}}\right) / K$. Suppose for some $c \in C, c \in K\left(F^{p}\right)\left(x^{p^{-e}}, C \backslash\{c\}\right)$. Then by the exchange property, $x^{p^{-e}} \in K\left(F^{p}\right)\left(x^{p^{-e+1}}, C\right)$. This contradicts the fact that the exponent of $x^{p^{-e}}$ over $F$ is $e$. Thus $C \cup\left\{x^{p^{-\varepsilon}}\right\}$ is a relative $p$-basis of $F\left(x^{p^{-\varepsilon}}\right) / K$.

COROLlary 8. In the notation of the introduction, $\operatorname{insep}(L / K)=d$ where $d$ is the number of $c_{i}$ such that $c_{i}^{p_{i}} \in K\left(L_{i-1}^{p}\right)$.

It is clear that, in the notation of the introduction where $L=D\left(c_{1}, \ldots, c_{m}\right), D$ being separable over $K$ is merely a matter of convenience. For example, if $D / K$ is inseparable, then Corollary 3 would simply be $\operatorname{inor}(L / K)=\operatorname{inor}(D / K)+$ $\sum_{i=1}^{m} \min \left\{e_{i}, r_{i}\right\}$. Thus, if $D$ is inseparable over $K, D / K$ is a form of $L / K$ if and only if each $r_{i}=0$. This shows how to construct extension with proper forms. Similarly, by a degree argument, every distinguished subfield of $D / K$ is one of $L / K$ if and only if $e_{i} \leqslant r_{i}$ for all $i$.

\section{REFERENCES}

1. J. Deveney and J. Mordeson, Subfields and invariants of inseparable field extensions, Canad. J. Math. 29 (1977), 1304-1311.

2. $\ldots$ The order of inseparability of fields, Canad. J. Math. 31 (1979), 655-662.

3. J. Dieudonné, Sur les extensions transcendantes separables, Summa Brasil. Math. 2 (1947), 1-20.

4. N. Heerema and J. Deveney, Galois theory for fields $K / \boldsymbol{k}$ finitely generated, Trans. Amer. Math. Soc. 189 (1974), 263-274.

5. N. Heerema, pth powers of distinguished subfields, Proc. Amer. Math. Soc. 55 (1976), 287-292.

6. H. Kraft, Inseparable Korpererweiterungen, Comment. Math. Helv. 45 (1970), 110-118.

7. A. Weil, Foundations of algebraic geometry, Amer. Math. Soc. Colloq. Publ., vol. 29, Amer. Math. Soc., Providence, R. I., 1946.

Department of Mathematical Sciences, Virginia Commonwealth University, Richmond, VIRGINIA 23284

Department of Mathematics, Creighton University, Omaha, Nebraska 68134 (Current address of J. N. Mordeson)

Current address (J. K. Deveney): Department of Mathematics, Louisiana State University, Baton Rouge, Louisiana 70803 